PROCEEDINGS OF THE

AMERICAN MATHEMATICAL SOCIETY

Volume 125, Number 12, December 1997, Pages 3481-3488

S 0002-9939(97)04045-8

\title{
INVARIANTS OF SKEW DERIVATIONS
}

\author{
JEFFREY BERGEN AND PIOTR GRZESZCZUK
}

(Communicated by Ken Goodearl)

\begin{abstract}
If $\sigma$ is an automorphism and $\delta$ is a $\sigma$-derivation of a ring $R$, then the subring of invariants is the set $R^{(\delta)}=\{r \in R \mid \delta(r)=0\}$. The main result of this paper is

Theorem. Let $\delta$ be a $\sigma$-derivation of an algebra $R$ over a commutative ring $K$ such that

$$
\delta^{n+k}(r)+a_{n-1} \delta^{n+k-1}(r)+\cdots+a_{1} \delta^{k+1}(r)+a_{0} \delta^{k}(r)=0,
$$

for all $r \in R$, where $a_{n-1}, \ldots, a_{1}, a_{0} \in K$ and $a_{0}{ }^{-1} \in K$.

(i) If $R^{n+1} \neq 0$, then $R^{(\delta)} \neq 0$.

(ii) If $L$ is a $\delta$-stable left ideal of $R$ such that l.ann $R(L)=0$, then $L^{(\delta)} \neq 0$.
\end{abstract}

This theorem generalizes results on the invariants of automorphisms and derivations.

If $R$ is a ring with an automorphism $\sigma$, we say that an additive map $\delta: R \longrightarrow R$ is a $\sigma$-derivation if

$$
\delta(r s)=\delta(r) s+\sigma(r) \delta(s)
$$

for all $r, s \in R$. We define the subring of invariants to be the set

$$
R^{(\delta)}=\{r \in R \mid \delta(r)=0\} .
$$

It was shown in $[\mathrm{HN}]$ that algebraic automorphisms always act with nonzero invariants on nonnilpotent algebras. The analogous result for algebraic derivations was proven in $[\mathrm{B}]$. The simplest examples of $\sigma$-derivations are ordinary derivations, which occur when $\sigma$ is the identity map, as well as maps of the form $1-\sigma$. Therefore the results in this paper generalize results on the invariants of automorphisms and derivations. However, the results on automorphisms and derivations were obtained using group-graded rings, whereas our arguments are entirely combinatorial. In fact, we will present an example in which the 0 -eigenspace of a $\sigma$-derivation is not a subring, thus the techniques of group-graded rings cannot be applied to this more general situation. Since we would like to apply our results to prove that various subrings and one-sided ideals contain nonzero invariants, we will not be assuming that our rings have a unit element.

Received by the editors December 29, 1995 and, in revised form, July 2, 1996.

1991 Mathematics Subject Classification. Primary 16W20, 16W25, 16W55.

The first author was supported by the University Research Council at DePaul University. Both authors were supported by Polish KBN Grant 2 PO3A 050 08. Much of this work was done when the first author was a visitor at the University of Warsaw, Białystok Division and the second author was a visitor at DePaul University. We would like to thank both universities for their hospitality.

(C)1997 American Mathematical Society 
We now define the terms we will be using throughout this paper. $R$ will be an algebra over a commutative ring $K$ and the automorphism $\sigma$ and $\sigma$-derivation $\delta$ will be assumed to be $K$-linear transformations. We will be assuming that $\delta$ is algebraic over $K$. By this we mean that

$$
\delta^{n+k}(r)+a_{n-1} \delta^{n+k-1}(r)+\cdots+a_{1} \delta^{k+1}(r)+a_{0} \delta^{k}(r)=0,
$$

for all $r \in R$, where $a_{n-1}, \ldots, a_{1}, a_{0} \in K$ and $a_{0}{ }^{-1} \in K$. We let $t: R \longrightarrow R$ be defined as

$$
t=\delta^{n}+\cdots+a_{1} \delta+a_{0} .
$$

Let

$$
R_{0}=\left\{r \in R \mid \delta^{m}(r)=0, \text { for some } m \geq 1\right\} ;
$$

since $a_{0}$ is invertible in $K$, a standard argument from linear algebra implies that the restriction $t: R_{0} \longrightarrow R_{0}$ is surjective. Therefore, it is now clear that

$$
t^{j}(R)=t(R)=R_{0}=\left\{r \in R \mid \delta^{k}(r)=0\right\},
$$

for all $j \geq 1$. If $k=1$, then we say that $\delta$ is separable and in this special case, $t$ maps $R$ onto $R^{(\delta)}$.

We should point out that we will be neither making any assumptions on whether $\sigma$ is algebraic, nor assuming that there exists any additional relationship between $\sigma$ and $\delta$. We will make use of the following notation: if $A, B, C$ are subsets of $R$ then $A B^{\#} C$ is the span over $K$ of the elements from the union of the sets $B, A B, B C$, and $A B C$. If $A$ is any subset of $R$, then we let $\operatorname{l.ann}_{R}(A)=\{r \in R \mid r A=0\}$. Subsets $B$ of $R$ with the properties that $\sigma(B)=B$ and $\delta(B) \subseteq B$ are known, respectively, as $\sigma$-stable and $\delta$-stable. Subsets satisfying both properties are called $(\sigma, \delta)$-stable. For any $A \subseteq R$, we let $A^{(\delta)}=A \cap R^{(\delta)}$. A ring with no nonzero nilpotent $(\sigma, \delta)$-stable ideals is called $(\sigma, \delta)$-semiprime and a ring with no nonzero nilpotent $\sigma$-stable ideals is called $\sigma$-semiprime. Note that in the special case where $\sigma$ is algebraic, semiprime and $\sigma$-semiprime are equivalent.

Lemma 1. For any $\delta$-stable left ideal $L$ of $R$,

$$
\sigma^{n}(L) \sigma^{n-1}(L) \cdots \sigma(L) L \subseteq R t(L)^{\#} L .
$$

Proof. Since $L$ is $\delta$-stable, $t(L)$ is a $\delta$-stable subspace of $L$. By the definition of $t$, it follows that if $x \in L$, then there exist $y \in L$ and $l \in t(L)$ such that

$$
x=\delta(y)+l .
$$

We will prove, by induction on $m$, that for any $0 \leq m \leq n$ and for every $x_{1}, \ldots, x_{m} \in L$, there exist $b_{0}, b_{1}, \ldots, b_{n-m-1} \in R$ such that

$$
\sigma^{n}\left(x_{1}\right) \sigma^{n-1}\left(x_{2}\right) \cdots \sigma^{n-m+1}\left(x_{m}\right) \delta^{n-m}(x)+\sum_{j=0}^{n-m-1} b_{j} \delta^{j}(x) \in R t(L)^{\#} L,
$$

for all $x \in L$.

Note that for every $x \in L$,

$$
\delta^{n}(x)+\sum_{j=0}^{n-1} a_{j} \delta^{j}(x)=t(x) \in R t(L)^{\#} L .
$$


Thus the $m=0$ case is done. Next, assume that $n>m \geq 0$ and, by the induction hypothesis, we may assume that $\left(^{*}\right)$ holds. If $x \in L$, let $y \in L$ such that $x=\delta(y)+l$, where $l \in t(L)$. Then replacing $x$ in $\left(^{*}\right)$ by $x_{m+1} y$, where $x_{m+1} \in L$, yields

$$
\begin{aligned}
& \left(\sigma^{n}\left(x_{1}\right) \sigma^{n-1}\left(x_{2}\right) \cdots \sigma^{n-m+1}\left(x_{m}\right) \delta^{n-m}\left(x_{m+1}\right)+\sum_{j=0}^{n-m-1} b_{j} \delta^{j}\left(x_{m+1}\right)\right) y \\
& \quad+\sum_{j=0}^{n-m-2} c_{j} \delta^{j+1}(y) \\
& \quad+\sigma^{n}\left(x_{1}\right) \sigma^{n-1}\left(x_{2}\right) \cdots \sigma^{n-m+1}\left(x_{m}\right) \sigma^{n-m}\left(x_{m+1}\right) \delta^{n-m}(y) \in R t(L)^{\#} L,
\end{aligned}
$$

for some $c_{j} \in R$.

By the induction hypothesis,

$$
\left(\sigma^{n}\left(x_{1}\right) \sigma^{n-1}\left(x_{2}\right) \cdots \sigma^{n-m+1}\left(x_{m}\right) \delta^{n-m}\left(x_{m+1}\right)+\sum_{j=0}^{n-m-1} b_{j} \delta^{j}\left(x_{m+1}\right)\right) y \in R t(L)^{\#} L,
$$

therefore

$$
\begin{gathered}
\sigma^{n}\left(x_{1}\right) \sigma^{n-1}\left(x_{2}\right) \cdots \sigma^{n-m+1}\left(x_{m}\right) \sigma^{n-m}\left(x_{m+1}\right) \delta^{n-m}(y) \\
+\sum_{j=0}^{n-m-2} c_{j} \delta^{j+1}(y) \in R t(L)^{\#} L .
\end{gathered}
$$

Replacing $\delta(y)$ by $x-l$ in $(* *)$, shows that

$$
\begin{aligned}
& \sigma^{n}\left(x_{1}\right) \sigma^{n-1}\left(x_{2}\right) \cdots \sigma^{n-m+1}\left(x_{m}\right) \sigma^{n-m}\left(x_{m+1}\right) \delta^{n-m-1}(x)+\sum_{j=0}^{n-m-2} c_{j} \delta^{j}(x) \\
& +\sigma^{n}\left(x_{1}\right) \sigma^{n-1}\left(x_{2}\right) \cdots \sigma^{n-m+1}\left(x_{m}\right) \sigma^{n-m}\left(x_{m+1}\right) \delta^{n-m-1}(l) \\
& \quad+\sum_{j=0}^{n-m-2} c_{j} \delta^{j}(l) \in R t(L)^{\#} L .
\end{aligned}
$$

However, $l \in t(L)$, and thus

$$
\begin{aligned}
& \sigma^{n}\left(x_{1}\right) \sigma^{n-1}\left(x_{2}\right) \cdots \sigma^{n-m+1}\left(x_{m}\right) \sigma^{n-m}\left(x_{m+1}\right) \delta^{n-m-1}(l) \\
& \quad+\sum_{j=0}^{n-m-2} c_{j} \delta^{j}(l) \in R t(L)^{\#} L .
\end{aligned}
$$

As a result, we now have

$$
\begin{aligned}
& \sigma^{n}\left(x_{1}\right) \sigma^{n-1}\left(x_{2}\right) \cdots \sigma^{n-m+1}\left(x_{m}\right) \sigma^{n-m}\left(x_{m+1}\right) \delta^{n-m-1}(x) \\
& +\sum_{j=0}^{n-m-2} c_{j} \delta^{j}(x) \in R t(L)^{\#} L .
\end{aligned}
$$

The proof of $(*)$ is now complete and the proof of the lemma follows by letting $m=n$ in $(*)$. 
We can now prove our first main result. It is worth noting that in part (ii) of the following theorem, we prove the existence of nonzero invariants in $L$ even though $L$ is not necessarily $\sigma$-stable.

Theorem 2. Let $\delta$ be a $\sigma$-derivation of an algebra $R$ over a commutative ring $K$ such that

$$
\delta^{n+k}(r)+a_{n-1} \delta^{n+k-1}(r)+\cdots+a_{1} \delta^{k+1}(r)+a_{0} \delta^{k}(r)=0,
$$

for all $r \in R$, where $a_{n-1}, \ldots, a_{1}, a_{0} \in K$ and $a_{0}{ }^{-1} \in K$.

(i) If $R^{n+1} \neq 0$, then $R^{(\delta)} \neq 0$.

(ii) If $L$ is a $\delta$-stable left ideal of $R$ such that l.ann $n_{R}(L)=0$, then $L^{(\delta)} \neq 0$.

Proof. For (i), let $L=R$ in Lemma 1. It then follows that if $R^{n+1} \neq 0$, then $t(R) \neq$ 0 . Since $\delta$ acts nilpotently on $t(R)$, it is clear that $R^{(\delta)} \neq 0$. For (ii), if $l$.ann $R(L)=$ 0 then $\operatorname{l.ann}_{R}\left(\sigma^{i}(L)\right)=0$, for all $i$. Therefore $\sigma^{n}(L) \sigma^{n-1}(L) \cdots \sigma(L) L \neq 0$. By Lemma 1, we have that $t(L) \neq 0$ and since $L$ is $\delta$-stable, it follows that $L^{(\delta)} \neq 0$.

We continue with

Lemma 3. Let $f(m)=\sum_{i=1}^{m}(n+1)^{i}$ and let $T=t(R)$. If $T$ is a subring of $R$ and if $T^{l}$ is $\delta$-stable, for all $l \geq 1$, then

$$
R^{f((m-1) k+1)} \subseteq R\left(T^{m}\right)^{\#} R,
$$

for any $m \geq 1$.

Proof. We proceed by induction on $m$. The $m=1$ case follows by letting $L=R$ in Lemma 1, as we have

$$
R^{f(1)}=R^{n+1} \subseteq R t(R)^{\#} R=R T^{\#} R .
$$

Now suppose that $m \geq 1$ such that

$$
R^{f((m-1) k+1)} \subseteq R\left(T^{m}\right)^{\#} R .
$$

Note that if $A$ is any $\delta$-stable subring of $R$, then

$$
t(R A) \subseteq t(R) A+R \delta(A)=T A+R \delta(A) .
$$

We now define a collection of $\delta$-stable left ideals as follows: $L_{0}=R T^{m}+T^{m}$ and $L_{j+1}=R t\left(R L_{j}\right)+t\left(R L_{j}\right)$, for $j \geq 0$. We claim that, for any $j \geq 1$,

$$
L_{j} \subseteq R T^{m+1}+T^{m+1}+R \delta^{j}\left(T^{m}\right) .
$$

We proceed by induction on $j$. For $j=1$, we have

$$
t\left(R L_{0}\right) \subseteq t\left(R T^{m}\right) \subseteq T^{m+1}+R \delta\left(T^{m}\right),
$$

and so,

$$
L_{1} \subseteq R T^{m+1}+T^{m+1}+R \delta\left(T^{m}\right) .
$$

Now let $j \geq 1 ;$ since $T^{m}$ and $T^{m+1}$ are $\delta$-stable, it follows by $(* * *)$ and the induction hypothesis on $j$ that

$$
\begin{aligned}
t\left(R L_{j}\right) & \subseteq t\left(R T^{m+1}+R \delta^{j}\left(T^{m}\right)\right) \\
& \subseteq T T^{m+1}+R \delta\left(T^{m+1}\right)+T \delta^{j}\left(T^{m}\right)+R \delta^{j+1}\left(T^{m}\right) \\
& \subseteq R T^{m+1}+T^{m+1}+R \delta^{j+1}\left(T^{m}\right) .
\end{aligned}
$$


Thus, we now have

$$
L_{j+1}=R t\left(R L_{j}\right)+t\left(R L_{j}\right) \subseteq R T^{m+1}+T^{m+1}+R \delta^{j+1}\left(T^{m}\right),
$$

as desired.

Next, we define a sequence of integers $g(j)$ as

$$
g(j)=(n+1)^{j} f((m-1) k+1)+\sum_{i=1}^{j}(n+1)^{i} .
$$

It then follows that $g(0)=f((m-1) k+1)$ and $g(j+1)=(g(j)+1)(n+1)$, for $j \geq 0$. We claim that

$$
R^{g(j)} \subseteq R L_{j}^{\#} R
$$

for $j \geq 0$ and we will proceed by induction on $j$. The $j=0$ case follows by the induction hypothesis on $m$ as

$$
R^{g(0)}=R^{f((m-1) k+1)} \subseteq R\left(T^{m}\right)^{\#} R \subseteq R L_{0}{ }^{\#} R .
$$

Now suppose that $j \geq 0$ such that $R^{g(j)} \subseteq R L_{j}{ }^{\#} R$ holds. By the induction hypothesis on $j$ and the surjectivity of $\sigma$, for all $i \geq 0$, we have

$\left(\right.$ ****) $\quad R^{g(j)+1}=R R^{g(j)}=R \sigma^{i}\left(R^{g(j)}\right) \subseteq R \sigma^{i}\left(R L_{j}^{\#} R\right) \subseteq R\left(R \sigma^{i}\left(L_{j}\right)^{\#} R\right)$.

It now follows from $(* * * *)$ and Lemma 1 that

$$
\begin{aligned}
\left(R^{g(j)+1}\right)^{n+1} & \\
& \subseteq\left(\sigma^{n}\left(R L_{j}\right) R+\sigma^{n}\left(R L_{j}\right)\right)\left(\sigma^{n-1}\left(R L_{j}\right) R+\sigma^{n-1}\left(R L_{j}\right)\right) \cdots\left(R L_{j} R+R L_{j}\right) \\
& \subseteq \sigma^{n}\left(R L_{j}\right) \sigma^{n-1}\left(R L_{j}\right) \cdots R L_{j} R+\sigma^{n}\left(R L_{j}\right) \sigma^{n-1}\left(R L_{j}\right) \cdots R L_{j} \\
& \subseteq R t\left(R L_{j}\right)^{\#} R \subseteq R L_{j+1}^{\#} R .
\end{aligned}
$$

Since $g(j+1)=(g(j)+1)(n+1)$, this implies that

$$
R^{g(j+1)} \subseteq R L_{j+1}^{\#} R,
$$

as desired.

As a result, for any $j \geq 0$, we have

$$
R^{g(j)} \subseteq R L_{j}^{\#} R \subseteq R\left(T^{m+1}\right)^{\#} R+R\left(\delta^{j}\left(T^{m}\right)\right)^{\#} R .
$$

Since $T$ is a subring, $\delta^{k}\left(T^{m}\right) \subseteq \delta^{k}(T)=0$. Therefore if we let $j=k$, we have

$$
R^{g(k)} \subseteq R\left(T^{m+1}\right)^{\#} R
$$

However, it is easy to see that $g(k)=f(m k+1)$, thus

$$
R^{f(m k+1)} \subseteq R\left(T^{m+1}\right)^{\#} R,
$$

as desired, thereby concluding the proof.

In Lemma 3, we assumed that $t(R)$ is a subring. We now give an example which shows that $t(R)$ need not be a subring. Since $t(R)$ is the 0 -eigenspace of $R$, this illustrates why it was necessary to prove the results in this paper without using group-graded rings. 
Example 4. A finite-dimensional simple ring $R$ with a $\sigma$-derivation $\delta$ such that $\delta^{4}=\delta^{2}$ and $\sigma^{2}=1$, but $t(R)$ is not a subring.

Let $S$ be a finite-dimensional simple ring with a noncentral idempotent $e$. Let $R=S_{2}$, the $2 \times 2$ matrices over $S$, and let $A=\left(\begin{array}{ll}0 & e \\ 0 & 0\end{array}\right)$ and $B=\left(\begin{array}{ll}0 & 1 \\ 1 & 0\end{array}\right)$. Next, let $\sigma$ be the inner automorphism of $R$ induced by $B$ and let $\delta$ be the inner $\sigma$-derivation of $R$ induced by $A$. More precisely, if $\left(\begin{array}{ll}r & s \\ t & u\end{array}\right) \in R$, then

$$
\begin{aligned}
\delta\left(\begin{array}{ll}
r & s \\
t & u
\end{array}\right) & =\left(\begin{array}{ll}
0 & e \\
0 & 0
\end{array}\right)\left(\begin{array}{ll}
r & s \\
t & u
\end{array}\right)-\left(\begin{array}{ll}
0 & 1 \\
1 & 0
\end{array}\right)\left(\begin{array}{ll}
r & s \\
t & u
\end{array}\right)\left(\begin{array}{ll}
0 & 1 \\
1 & 0
\end{array}\right)\left(\begin{array}{ll}
0 & e \\
0 & 0
\end{array}\right) \\
& =\left(\begin{array}{cc}
e t & e u-u e \\
0 & -s e
\end{array}\right) .
\end{aligned}
$$

If we let $d$ denote the inner derivation of $S$ induced by $e$, then we note that $d^{2}(s) e=-d(s) e$, for all $s \in S$. Therefore, it is easy to see that

$$
\delta\left(\begin{array}{cc}
r & s \\
t & u
\end{array}\right)=\left(\begin{array}{cc}
e t & d(u) \\
0 & -s e
\end{array}\right), \delta^{2}\left(\begin{array}{cc}
r & s \\
t & u
\end{array}\right)=\left(\begin{array}{cc}
0 & -d(s) e \\
0 & -d(u) e
\end{array}\right),
$$

and

$$
\delta^{4}\left(\begin{array}{ll}
r & s \\
t & u
\end{array}\right)=\left(\begin{array}{ll}
0 & d^{2}(s) e \\
0 & d^{2}(u) e
\end{array}\right)=\left(\begin{array}{cc}
0 & -d(s) e \\
0 & -d(u) e
\end{array}\right) .
$$

As a result, it is clear that $\delta^{4}=\delta^{2}$ and $\sigma^{2}=1$. We can observe that

$$
t(R)=\left\{\left(\begin{array}{cc}
r & s \\
t & u
\end{array}\right) \in R \mid d(s) e=d(u) e=0\right\} .
$$

Since $S$ is simple, there exist $s_{1}, s_{2} \in S$ such that $(e-1) s_{1} e s_{2} e \neq 0$. Furthermore, since $d\left(e s_{2}\right) e=0$, it follows that

$$
\left(\begin{array}{cc}
s_{1} & 0 \\
0 & 0
\end{array}\right),\left(\begin{array}{cc}
0 & e s_{2} \\
0 & 0
\end{array}\right) \in t(R) .
$$

However,

$$
\left(\begin{array}{cc}
s_{1} & 0 \\
0 & 0
\end{array}\right)\left(\begin{array}{cc}
0 & e s_{2} \\
0 & 0
\end{array}\right)=\left(\begin{array}{cc}
0 & s_{1} e s_{2} \\
0 & 0
\end{array}\right)
$$

and $d\left(s_{1} e s_{2}\right) e=(e-1) s_{1} e s_{2} e \neq 0$. Thus

$$
\left(\begin{array}{cc}
0 & s_{1} e s_{2} \\
0 & 0
\end{array}\right) \notin t(R)
$$

and so, $t(R)$ is not a subring.

In the next lemma we will show that if $t(R)$ is $\sigma$-stable, then $t(R)$ must be a subring. In this case, it easily follows that $t(R)^{l}$ is $\delta$-stable, for all $l \geq 1$, thus the hypotheses of Lemma 3 are satisfied. One large class of $\sigma$-derivations with the property that $t(R)$ is $\sigma$-stable is $q$-skew derivations. A $q$-skew derivation is a $\sigma$-derivation with the property that there exists some invertible $q \in K$ such that $\delta \sigma=q \sigma \delta$.

Lemma 5. If $t(R)$ is $\sigma$-stable, then $t(R)$ is a subring of $R$. 
Proof. Let $r, s \in t(R)$; then we have

$$
\delta^{k^{2}}(r s)=\sum_{i=0}^{k^{2}} F_{i, \delta, \sigma}(r) \delta^{i}(s),
$$

where $F_{i, \delta, \sigma}$ is a noncommutative polynomial in $\delta$ and $\sigma$. Each monomial in $F_{i, \delta, \sigma}$ is of degree $k^{2}$ such that $\delta$ appears $k^{2}-i$ times and $\sigma$ appears $i$ times. Therefore, whenever $i<k$, every monomial in $F_{i, \delta, \sigma}$ must contain a string in which $\delta$ appears at least $k$ consecutive times. Since $t(R)$ is stable under both $\sigma$ and $\delta$ and $\delta^{k}(t(R))=0$, it now follows that $F_{i, \delta, \sigma}(r)=0$, for all $i<k$. However, since $\delta^{i}(s)=0$, for all $i \geq k$, we see that $F_{i, \delta, \sigma}(r) \delta^{i}(s)=0$, for all $i \leq k^{2}$. Thus $\delta^{k^{2}}(r s)=0$ and so, $r s \in t(R)$.

We can now prove our second main result.

Theorem 6. Let $\delta$ be a $\sigma$-derivation of an algebra $R$ over a commutative ring $K$ such that $t(R)$ is $\sigma$-stable and

$$
\delta^{n+k}(r)+a_{n-1} \delta^{n+k-1}(r)+\cdots+a_{1} \delta^{k+1}(r)+a_{0} \delta^{k}(r)=0,
$$

for all $r \in R$, where $a_{n-1}, \ldots, a_{1}, a_{0} \in K$ and $a_{0}{ }^{-1} \in K$.

(i) If $t(R)$ is nilpotent, then $R$ is nilpotent. In particular, if $t(R)^{m}=0$ then

$$
R^{f((m-1) k+1)}=0 \text {, where } f(m)=\sum_{i=1}^{m}(n+1)^{i} .
$$

(ii) If $R$ is $(\sigma, \delta)$-semiprime, then $t(R)$ is $(\sigma, \delta)$-semiprime.

Proof. Since $t(R)$ is $\sigma$-stable, it follows by Lemma 5 that $t(R)$ satisfies the hypotheses of Lemma 3. The proof of (i) now follows immediately from Lemma 3. For (ii), if $t(R)$ is not $(\sigma, \delta)$-semiprime, then $t(R)$ contains a $(\sigma, \delta)$-stable ideal $I \neq 0$ such that $I^{2}=0$. Therefore $R I$ is a $(\sigma, \delta)$-stable left ideal of $R$ and so, $R I$ is not nilpotent. Hence, by Lemma $3, t(R I)$ is also not nilpotent. However, by $\left({ }^{* * *}\right)$ in the proof of Lemma 3,

$$
t(R I) \subseteq t(R) I+R \delta(I) \subseteq I+R \delta(I) .
$$

Since $I$ is $\delta$-stable, continuing in the manner, we see that

$$
t(R I)=t^{j}(R I) \subseteq I+R \delta^{j}(I),
$$

for all $j \geq 1$. Recall that $\delta^{k}(I) \subseteq \delta^{k}(t(R))=0$; therefore, by letting $j=k$, it follows that

$$
t(R I)=t^{k}(R I) \subseteq I .
$$

Thus

$$
(t(R I))^{2} \subseteq I^{2}=0,
$$

which contradicts the fact that $t(R I)$ is not nilpotent. Thus $t(R)$ is $(\sigma, \delta)$-semiprime.

For the special case where $\delta$ is separable, we can sharpen Theorem 6 and we record this as 
Corollary 7. Let $\delta$ be a separable $\sigma$-derivation of a ring $R$; that is,

$$
\delta^{n+1}(r)+a_{n-1} \delta^{n}(r)+\cdots+a_{1} \delta^{2}(r)+a_{0} \delta(r)=0,
$$

for all $r \in R$, where $a_{n-1}, \ldots, a_{1}, a_{0} \in K$ and $a_{0}{ }^{-1} \in K$.

(i) If $R^{(\delta)}$ is nilpotent, then $R$ is nilpotent. In particular, if $\left(R^{(\delta)}\right)^{m}=0$ then

$$
R^{f(m)}=0, \text { where } f(m)=\sum_{i=1}^{m}(n+1)^{i} .
$$

(ii) If $R$ is $(\sigma, \delta)$-semiprime and if $R^{(\delta)}$ is $\sigma$-stable, then $R^{(\delta)}$ is $\sigma$-semiprime.

Proof. Since $R^{(\delta)}=t(R)$ is a subring and $\left(R^{(\delta)}\right)^{l}$ is $\delta$-stable, for all $l \geq 1$, the proof of (i) follows directly from Lemma 3 with $k=1$. We now observe that $R^{(\delta)}$ is clearly $(\sigma, \delta)$-semiprime if and only if it is $\sigma$-semiprime. Therefore, (ii) is merely the special case of Theorem 6 (ii) with $k=1$.

We conclude this paper with an example which shows that the hypothesis in Corollary 7 (ii) that $R^{(\delta)}$ be $\sigma$-stable is necessary.

Example 8. A finite-dimensional simple ring $R$ with a $\sigma$-derivation $\delta$ such that $\delta^{2}=\delta$ and $\sigma^{2}=1$, but $R^{(\delta)}$ is not semiprime.

Let $S$ be a finite-dimensional simple ring and let $R=S_{2}$, the $2 \times 2$ matrices over $S$. Let $A=\left(\begin{array}{ll}0 & 0 \\ 0 & 1\end{array}\right)$ and $B=\left(\begin{array}{ll}0 & 1 \\ 1 & 0\end{array}\right)$ and then let $\sigma$ be the inner automorphism of $R$ induced by $B$ and let $\delta$ be the inner $\sigma$-derivation of $R$ induced by $A$. More precisely, if $\left(\begin{array}{ll}r & s \\ t & u\end{array}\right) \in R$, then

$$
\delta\left(\begin{array}{ll}
r & s \\
t & u
\end{array}\right)=\left(\begin{array}{ll}
0 & 0 \\
0 & 1
\end{array}\right)\left(\begin{array}{ll}
r & s \\
t & u
\end{array}\right)-\left(\begin{array}{ll}
0 & 1 \\
1 & 0
\end{array}\right)\left(\begin{array}{ll}
r & s \\
t & u
\end{array}\right)\left(\begin{array}{ll}
0 & 1 \\
1 & 0
\end{array}\right)\left(\begin{array}{ll}
0 & 0 \\
0 & 1
\end{array}\right)=\left(\begin{array}{cc}
0 & -t \\
t & u-r
\end{array}\right) .
$$

It is easy to see that $\delta^{2}=\delta$ and $\sigma^{2}=1$. In addition, we can now observe that

$$
R^{(\delta)}=\left\{\left(\begin{array}{cc}
r & s \\
0 & r
\end{array}\right) \mid r, s \in S\right\}
$$

Since $\left(\begin{array}{ll}0 & 1 \\ 0 & 0\end{array}\right) \in R^{(\delta)}$ and

$$
\left(\begin{array}{ll}
0 & 1 \\
0 & 0
\end{array}\right) R^{(\delta)}\left(\begin{array}{ll}
0 & 1 \\
0 & 0
\end{array}\right)=0
$$

we see that $R^{(\delta)}$ is not semiprime.

\section{REFERENCES}

[B] J. Bergen, Constants of Lie algebra actions, J. Algebra 114 (1988), 452-465. MR 89c:16048 [HN] I.N. Herstein and L. Neumann, Centralizers in rings, Ann. Mat. Pura Appl. 102 (1975), 37-44. MR 50:13143

Department of Mathematics, DePaul University, Chicago, Illinois 60614

E-mail address: jbergen@condor.depaul.edu

Institute of Mathematics, University of Warsaw, Bialystok Division Akademicka 2, 15-267, BiaŁystok, Poland

E-mail address: piotrgr@cksr.ac.bialystok.pl 